\title{
Effective Properties of Semitransparent Radiative Cooling Materials With Spectrally Variable Properties
}

\author{
Ravi Anant Kishore ( $\square$ ravi.kishore@nrel.gov) \\ National Renewable Energy Laboratory \\ Chuck Booten \\ National Renewable Energy Laboratory \\ Sajith Wijesuriya \\ National Renewable Energy Laboratory
}

\section{Research Article}

Keywords: Radiation cooling, spectral variable properties, effective properties, semitransparent coating, participating substrate.

Posted Date: August 5th, 2021

DOl: https://doi.org/10.21203/rs.3.rs-777647/v1

License: (c) (i) This work is licensed under a Creative Commons Attribution 4.0 International License. Read Full License

Version of Record: A version of this preprint was published at Applied Thermal Engineering on January 1st, 2022. See the published version at https://doi.org/10.1016/j.applthermaleng.2022.118048. 


\title{
Effective Properties of Semitransparent Radiative Cooling Materials With Spectrally Variable Properties
}

\author{
Ravi Anant Kishore, ${ }^{+, *}$ Chuck Booten ${ }^{+, *}$, and Sajith Wijesuriya \\ National Renewable Energy Laboratory, 15013 Denver West Parkway, Golden, CO 80401.
}

\begin{abstract}
Radiation cooling is a promising solid-state, non-vapor-compression technology for passive refrigeration and air conditioning. Although this phenomenon occurs naturally, achieving a significant amount of cooling to make it a technically and economically viable technology requires highly engineered, spectrally selective radiative surfaces. These characteristics make radiation cooling difficult to estimate, particularly when it is integrated with other systems such as photovoltaic panels or building envelopes. The complexity further increases when the substrate also participates in the radiative cooling (along with the radiative coating). Energy estimation is becoming increasingly critical because of the recent focus on the semitransparent radiative coatings that transmit a variety of colors to enhance the aesthetic appeal of the system. Here, we propose a simple iterative method to calculate the effective radiative properties, which provide the same net radiative cooling that would be observed using the spectral properties at both the coating and substrate surfaces. Compared to traditional methods that rely on either computationally expensive full spectral analysis or methods for averaging each radiative surface parameter locally, our proposed method focuses on calculating effective properties that provide the same the net cooling effect as a full spectral analysis by accounting the emissivity, absorptivity, and transmissivity collectively, thereby providing an overall estimation error of less than $0.2 \%$. We believe that this study will be beneficial to the engineering communities that employ complex simulation codes and require lumped solar and thermal radiation related parameters.

Keywords: Radiation cooling, spectral variable properties, effective properties, semitransparent coating, participating substrate.

+ These authors contributed equally to the work.

* Corresponding authors: ravi.kishore@ nrel.gov (RAK) and Chuck.Booten@nrel.gov (CB)
\end{abstract}




\section{Introduction}

Radiative sky cooling (aka radiative terrestrial cooling or radiation cooling) is gaining tremendous interest as an effective cooling technique and a promising alternative for traditional vaporcompression air conditioners that typically consume large amount of electricity and use refrigerants with high global warning potential. ${ }^{1}$ Radiative cooling is an important natural mechanism that enables the earth to maintain its temperature by balancing heat gain from the sun and heat loss to space. Because it is a natural process, it does not require any external input energy for operation. However, it requires a combination of solar and thermal surface properties that may not be prominently present in bulk materials, making it difficult to observe. ${ }^{2}$

Radiative cooling, in principle, is based on a natural phenomenon that allows the atmosphere to transmit about $87 \%$ of the outgoing long-wavelength radiation from the earth in an infrared region of 8-13 $\mu \mathrm{m}$, called the sky or atmospheric window. ${ }^{3}$ It has been reported that certain spectrally selective coatings, such as organic polymers, ${ }^{4,5}$ titanium dioxide, ${ }^{6,7}$ silicon nitrides, ${ }^{8}$ and silicon monoxide, ${ }^{9}$ that emit strongly in the atmospheric window region can provide on average 40-80 $\mathrm{W} / \mathrm{m}^{2}$ of net cooling flux ${ }^{10,11}$ and surface temperature drop up to $10-15^{\circ} \mathrm{C}$ below ambient at night with clear sky conditions. ${ }^{12,13}$ The cooling capacity and operating time can be substantially improved by combining nighttime radiation cooling with daytime radiation cooling that requires materials with extremely high reflectivity in the highly intensive solar spectrum wavelength (0.3$2.5 \mu \mathrm{m})$ while having high emissivity in the atmospheric window $(8-13 \mu \mathrm{m})$. Recently developed metal-dielectric photonic surfaces that behave as a broadband mirrors for solar light and simultaneously emit strongly in the atmospheric transparency window can theoretically achieve a net cooling power exceeding $100 \mathrm{~W} / \mathrm{m}^{2}$ at ambient temperature. ${ }^{14,15}$

With significant recent research and development in materials science, radiation cooling technology is becoming increasingly effective, deployable, and practical. It has been proposed to be used for several engineering applications such as photovoltaic appliances, electronic cooling, infrared detectors, and other temperature-sensitive sensors. ${ }^{2}$ Buildings are one of the most targeted applications of this technology due to its notable impact on reducing the energy use for air conditioning. Radiative coolers in the form of either standalone cooling panels or cooling coatings for envelope components (such as the roof) can reduce HVAC energy use as well as reduce the peaks in the cooling load profile that typically occur during the daytime. ${ }^{16}$ 
As the application areas increase, so is the need to correctly model spectrally distributed radiation properties (absorptivity, transmissivity, reflectivity, and emissivity) of radiation coatings. Although the actual materials exhibit spectrally dependent properties, it is practically convenient if radiative parameters can be expressed in terms of a constant lumped or effective value. As an example, whole-building energy simulation program EnergyPlus ${ }^{\circledR}$ requires a constant value of solar absorptivity and thermal emissivity for the opaque envelope. Clearly, accurate calculation of the effective radiation properties is critical as their inaccurate estimation can result in incorrect predictions as well as faulty system design, leading to engineering failures.

In this study, we formulate and validate an iterative method to estimate the effective radiative properties of a semitransparent coating with spectrally distributed surface properties. Compared to the traditional method, which relies on averaging each of the radiation-related parameters separately, we focus on the net radiation heat flux comprising absorptance, emittance, and transmittance collectively, thereby minimizing the error in the net radiation cooling calculations. We further compare the proposed method using a commercial finite element code, COMSOL Multiphysics, and note a significant improvement over the traditional method. This study will be particularly beneficial for engineering simulations like EnergyPlus that employ complex numerical codes and require lumped solar and thermal radiation related parameters.

\section{Methods}

\subsection{Radiative Cooling by an Opaque, Diffuse, and Gray Coating}

As shown in Figure 1(a), the net cooling power $\left(Q_{n e t}\right)$ generated by an opaque radiative coating surface can be expressed as: ${ }^{15}$

$Q_{\text {net }}\left(T_{s}\right)=Q_{\text {rad }}\left(T_{s}\right)-Q_{\text {sky }}\left(T_{\text {sky }}\right)-Q_{\text {solar }}-Q_{\text {gain }}$

where $Q_{\text {rad }}$ is the total thermal radiation emitted from the surface at temperature $T_{S}$; this includes radiation to the sky as well as radiation to space. $Q_{s k y}$ is the absorbed radiation from the sky at temperature $T_{s k y}$ on the surface (the assumption is that the view factor between the surface and the sky is unity), $Q_{\text {solar }}$ is the absorbed solar irradiation on the surface, and $Q_{\text {gain }}$ is the convectionand conduction-related heat gain. 

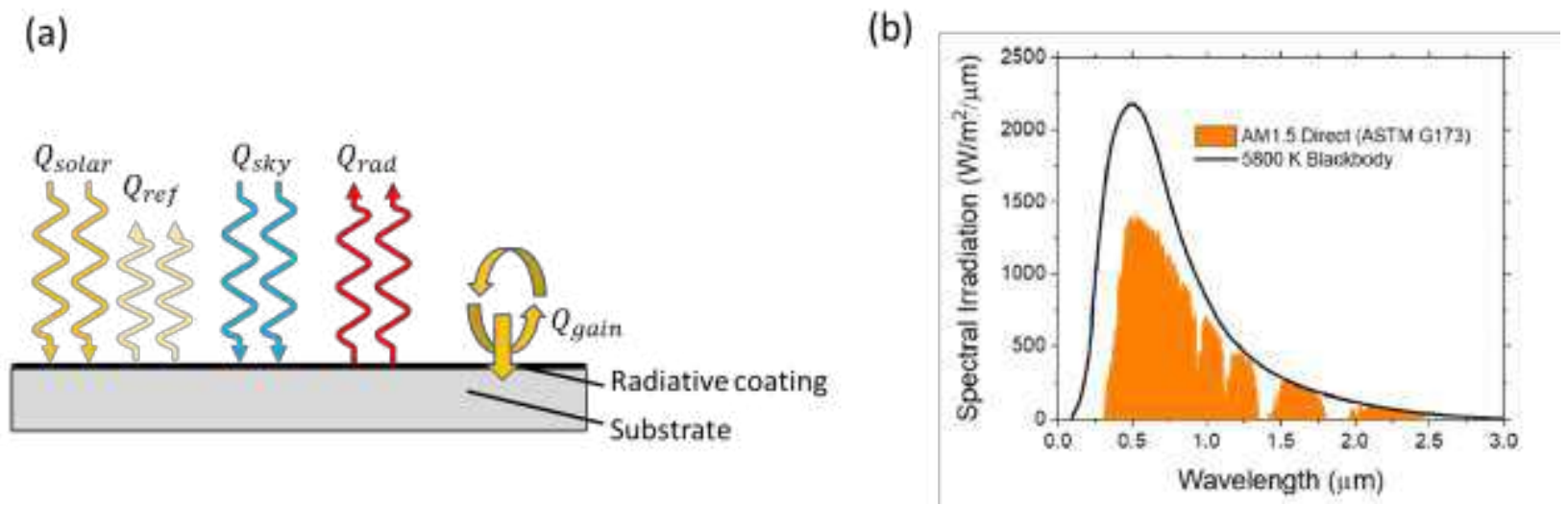

Figure 1. Radiative cooling: (a) heat transfer processes at an opaque radiative coating surface, (b) solar irradiance on a horizontal surface at air mass (AM) coefficient 1.5 and blackbody spectrum at the solar temperature of $5,800 \mathrm{~K} .^{1}$

Thermal radiation, $Q_{\text {rad }}$, from a gray surface that emits in all directions is associated with a hypothetical hemisphere surround the surface and is characterized by a directional distribution expressed as: ${ }^{17}$

$Q_{\text {rad }}\left(T_{s}\right)=A_{s} \int_{0}^{\infty} \int_{0}^{\pi / 2} \int_{0}^{2 \pi} \varepsilon_{s}(\lambda, \theta, \phi) I_{b b}\left(T_{s}, \lambda, \theta, \phi\right) \cos \theta \sin \theta d \lambda d \theta d \phi$

where $A_{s}$ is the surface area, $T_{s}$ is the surface temperature, $\lambda$ is the radiation wavelength, $\theta$ is the zenith or polar angle, and $\phi$ is the azimuthal angle of the hemisphere. $\varepsilon_{S}(\lambda, \theta, \phi)$ is the emissivity of the surface, and $I_{b b}(T, \lambda)$ is the spectral radiance of a blackbody, which is expressed according to Planck's law as ${ }^{18}$ :

$I_{b b}(T, \lambda)=\frac{2 h c^{2}}{\lambda^{5}} \frac{1}{\exp \left(h c / \lambda k_{B} T\right)-1}$

where $h$ and $k_{B}$ are the universal Planck and Boltzmann constants, and $c$ is the speed of light in a vacuum.

When the radiative surface is diffuse, the emission occurs uniformly in all directions. Therefore, the radiation term can be simplified as:

$Q_{\text {rad }}\left(T_{s}\right)=\pi A_{s} \int_{0}^{\infty} \varepsilon_{s}(\lambda) I_{b b}\left(T_{s}, \lambda\right) d \lambda$

Combining equations (3) and (4), we can write:

$Q_{\text {rad }}\left(T_{s}\right)=A_{s} \int_{0}^{\infty} \varepsilon_{s}(\lambda) \frac{C_{1}}{\lambda^{5}} \frac{1}{\exp \left(C_{2} / T_{s} \lambda\right)-1} d \lambda$ 
where $C_{1}=2 \pi h c^{2}$ and $C_{2}=h c / k_{B}$.

Likewise, thermal radiation from the sky absorbed by the radiative surface can be given as:

$Q_{s k y}\left(T_{s k y}\right)=A_{s} \int_{0}^{\infty} \varepsilon_{s k y}(\lambda, \theta) \varepsilon_{s}(\lambda) \frac{C_{1}}{\lambda^{5}} \frac{1}{\exp \left(C_{2} / T_{s k y} \lambda\right)-1} d \lambda$

where $\varepsilon_{s k y}(\lambda, \theta)$ is the spectral directional emissivity of the sky, which can be expressed in terms of spectral emissivity in the zenith direction as ${ }^{19}$ :

$\varepsilon_{s k y}(\lambda, \theta)=1-\left(1-\varepsilon_{s k y}(\lambda, 0)\right)^{1 / \cos \theta}$

where the terms $1-\varepsilon_{s k y}(\lambda, 0)=\tau_{s k y}(\lambda, 0)$ is the sky transmittance in the zenith direction, which is typically highest in the wavelength interval 8-13 $\mu \mathrm{m}$ (atmospheric window), but it is affected by local conditions, cloud cover, and air humidity.

Lastly, the incident solar power absorbed by the surface is given as: ${ }^{20}$

$Q_{\text {solar }}=A_{s} \int_{0}^{\infty} \varepsilon\left(\lambda, \theta_{\text {sun }}\right) I_{\text {solar }}(\lambda) d \lambda$

where $\theta_{\text {sun }}$ is the angle between the incident direction of solar irradiation and the normal direction of the surface, and $I_{\text {solar }}(\lambda)$ is the wavelength-dependent direct solar irradiance. Figure 1(b) illustrates the spectral distribution of solar irradiation under the air mass (AM) coefficient of 1.5. ${ }^{1}$ The AM coefficient is expressed as the direct optical path length through the earth's atmosphere relative to the path length vertically upward in the zenith direction.

The additional term, $Q_{\text {gain }}$, accounts for conduction- and convection-related heat gain, and it may or may not be negligible depending upon the application. Conduction gains can be minimized by insulating the substrate from the bottom, whereas convection gains can be reduced by convection inhibitors or convection shields, which are typically radiatively transparent across a broad range of wavelengths. A common example of convection shield is the polyethylene film placed on top of the radiative surface maintaining a small air gap. Polyethylene film with a thickness of about $10 \mu \mathrm{m}$ has been reported to exhibit an average radiation transmission above $90 \%$ in the $8-13 \mu \mathrm{m}$ wavelengths range. ${ }^{21}$

\subsection{Traditional Effective Properties}

The effective radiative surface properties are defined as an average parameter that provides the same total radiation power as the spectral properties over all wavelengths. Traditionally, each of 
the surface properties are calculated independent of others. For instance, the effective thermal emissivity is expressed as: ${ }^{1}$

Effective thermal emissivity $\bar{\varepsilon}=\frac{\int_{0}^{\infty} \varepsilon_{S}(\lambda) \frac{C_{1}}{\lambda^{5} \exp \left(C_{2} / T \lambda\right)-1} d \lambda}{\sigma T_{s}^{4}}$

Likewise, the effective solar absorptivity and reflectivity are calculated as: ${ }^{22}$

Effective solar absorptivity $\bar{\alpha}=\frac{\int_{0}^{\infty} I_{\text {solar }}(\lambda) \varepsilon(\lambda) d \lambda}{\int_{0}^{\infty} I_{\text {solar }}(\lambda) d \lambda}$

Effective reflectivity $\bar{r}=\frac{\int_{0}^{\infty} I_{\text {solar }}(\lambda) r(\lambda) d \lambda}{\int_{0}^{\infty} I_{\text {solar }}(\lambda) d \lambda}$

Such that,

$Q_{\text {solar }}=\bar{\alpha} A_{s} Q_{\text {solar }}^{\text {total }}$, and

$Q_{\text {rad }}\left(T_{s}\right)=\bar{\varepsilon} \sigma A_{s} T_{s}^{4}$

where $Q_{\text {solar }}^{\text {total }}$ in the total solar power on the surface, and $\sigma$ is the Stefan-Boltzmann constant (5.67 $\left.\times 10^{-8} \mathrm{Wm}^{-2} \mathrm{~K}^{-4}\right)$.

Equations (9) to (13) suffice when the emitting surface has relatively constant radiative properties in solar and thermal regimes. Also, because transmissivity is not accounted for, it is assumed that the radiative coating is opaque and thus the substrate does not participate in the radiation cooling. In most practical cases, however, the radiative coating is thin and semitransparent; therefore, substrate participates in the radiation, and the radiation at the substrate surface as well as indirect radiation due to internal transmittance and reflections cannot be ignored.

\subsection{Semitransparent Coating With Participating Substrate}

For a participating substrate, Equation 1 becomes more complex. We need to account for the direct as well as indirect radiations due to transmission through the radiative coating and reflections between the outer (coating) and inner (substrate) surfaces. Figure 2 shows the heat transfer processes when the radiative cooling surface is thin and semitransparent, and the substrate surface is opaque and participating.

$Q_{\text {net }}(T)=\sum Q_{\text {rad }}(T)-\sum Q_{\text {sky }}\left(T_{\text {sky }}\right)-\sum Q_{\text {solar }}-Q_{\text {gain }}$ 
Although all the terms remain unchanged from equation (1), the summation $(\Sigma)$ in equation (14) represents the total, comprising both direct and indirect radiations at the outer and inner surfaces.

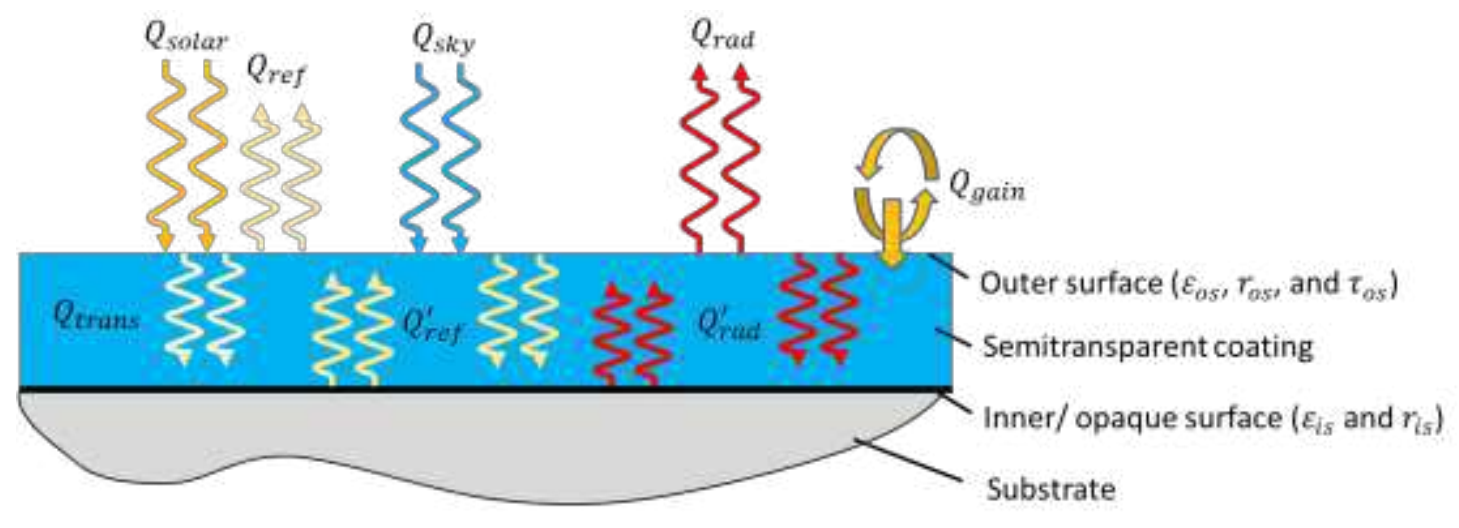

Figure 2. Heat transfer processes at the semitransparent radiative coating with a participating substrate. Note only first reflection and transmission are shown here. In practice, the radiations in a semitransparent radiative coating undergo multiple subsequent reflections and transmissions between the outer and inner surfaces.

To simplify the calculations, we assume the radiative cooling layer is thin, that all absorbed radiation is at the surface, and that it does not scatter the radiation (the medium is nonparticipating). Further, we assume that the radiative properties are dependent only on the wavelength and are independent of the angle of incidence relative to the surface and the temperature of the material in the range of operating temperatures. Lastly, we assume that the radiative properties at the two sides of coating material are the same.

As shown in Figure 2, we define radiative terms for the outer (coating) and inner (substrate) surfaces: $\varepsilon_{o S}(\lambda), r_{o S}(\lambda)$, and $\tau_{o S}(\lambda)$ as the wavelength-dependent emissivity, reflectivity, and transmissivity of the outer surface; $\varepsilon_{i s}(\lambda)$ and $r_{i s}(\lambda)$ as the wavelength-dependent emissivity and reflectivity of the inner surface; and $\varepsilon_{s k y}(\lambda)$ as the wavelength-dependent emissivity of the sky.

To account for radiation transmitted internally between the two surfaces, we define a term called transmittance. Under the assumptions that irradiance is unity and beam to diffuse reflectivity and transmissivity for the coating and the substrate are negligible, the transmittance can be mathematically expressed as: ${ }^{23}$ 
$\Gamma(\lambda)=\frac{\tau_{o S}(\lambda)}{1-r_{o s}(\lambda) r_{i s}(\lambda)}$

The net solar radiation absorbed by the outer surface can now be given as:

$Q_{o, \text { solar }}=A_{s} \int_{0}^{\infty} I_{\text {solar }}(\lambda) \varepsilon_{\text {os }}(\lambda) d \lambda+A_{s} \int_{0}^{\infty} I_{\text {solar }}(\lambda) \Gamma(\lambda) r_{\text {is }}(\lambda) \varepsilon_{\text {os }}(\lambda) d \lambda$

Note the first term in equation (16) accounts for the direct solar irradiance absorbed by the outer surface, whereas the second term represents the absorbed indirect solar irradiance that is transmitted and reflected between the two surfaces. The net solar radiation absorbed by the outer surface, $Q_{o, \text { solar }}$, can be simplified as:

$Q_{o, \text { solar }}=A_{s} \int_{0}^{\infty} I_{\text {solar }}(\lambda) \varepsilon_{o s}(\lambda)\left(1+\Gamma(\lambda) r_{i s}(\lambda)\right) d \lambda$

Likewise, we can express the solar irradiance absorbed by the inner surface as:

$Q_{i, \text { solar }}=A_{s} \int_{0}^{\infty} I_{\text {solar }}(\lambda) \varepsilon_{i s}(\lambda) \Gamma(\lambda) d \lambda$

The net radiation exchange between the outer surface and the sky is:

$Q_{\text {rad }}^{o s}\left(T_{o s}, T_{s k y}\right)=A_{s} \int_{0}^{\infty} \varepsilon_{o s}(\lambda)\left(1+\Gamma(\lambda) r_{i s}(\lambda)\right) \frac{C_{1}}{\lambda^{5}}\left[\frac{1}{\exp \left(C_{2} / T_{o s} \lambda\right)-1}-\frac{\varepsilon_{s k y}(\lambda)}{\exp \left(C_{2} / T_{s k y} \lambda\right)-1}\right] d \lambda$

Lastly, the thermal radiation exchanged between the inner surface and the sky can be given as:

$Q_{\text {rad }}^{i s}\left(T_{i s}, T_{\text {sky }}\right)=A_{s} \int_{0}^{\infty} \varepsilon_{i s}(\lambda) \Gamma(\lambda) \frac{C_{1}}{\lambda^{5}}\left[\frac{1}{\exp \left(C_{2} / T_{i s} \lambda\right)-1}-\frac{\varepsilon_{s k y}(\lambda)}{\exp \left(C_{2} / T_{s k y} \lambda\right)-1}\right] d \lambda$

Because the radiative coating is thin, the outer and inner surface temperatures are nearly the same, and thus the radiation exchange between the two surfaces can be neglected:

$Q_{\text {rad }}^{i o}\left(T_{o s}, T_{i s}\right) \approx 0$

\subsection{Effective Properties in the Solar and Thermal Region}

For radiation cooling calculations, the radiation spectrum is typically divided into two regions: solar and thermal. The solar region is the band of wavelength where solar power is mostly concentrated $(0.3-2.4 \mu \mathrm{m})$, whereas the thermal region contains wavelengths at which the radiative surface emits, which is 5-35 $\mu \mathrm{m}$ for the blackbody at around $300 \mathrm{~K}$. In this study, we considered $\lambda_{o}=2.5 \mu \mathrm{m}$ as the critical wavelength dividing the solar and thermal regions, and define the 
effective solar and thermal radiative surface properties to be the values that provide the same net cooling $\left(Q_{n e t}\right)$ that would be observed using the spectral calculations for both the coating and substrate. Because the surface temperature in a radiative cooling application is typically near 300 $\pm 20 \mathrm{~K}, \lambda_{o}=2.5 \mu \mathrm{m}$ is the commonly used cut-off wavelength between solar and thermal regions; however, the derivations provided is this study are generic and could be used for any $\lambda_{o}$ based on the application and the temperature of the radiating surface.

We express effective properties as follows: $\bar{\alpha}_{\text {solar }}^{\text {os }}$ as the effective solar absorptivity of the outer surface, $\bar{\varepsilon}_{\text {thermal }}^{\text {os }}$ as the effective thermal emissivity of the outer surface, $\bar{\alpha}_{\text {solar }}^{\text {is }}$ as the effective solar absorptivity of the inner surface, and $\bar{\varepsilon}_{\text {thermal }}$ as the effective thermal emissivity of the inner surface. Likewise, the effective reflectivity of the outer and inner surfaces in solar and thermal regions are denoted as $\bar{r}_{\text {solar }}^{\text {os }}, \bar{r}_{\text {thermal }}^{\text {os }}$ and $\bar{r}_{\text {solar }}^{\text {is }}, \bar{r}_{\text {thermal }}^{\text {is }}$, respectively. Lastly, the effective transmissivity of the outer layer is represented as $\bar{\tau}_{\text {soalr }}^{\text {os }}$ and $\bar{\tau}_{\text {thermal }}^{\text {os }}$.

Using Equation (15), the effective transmittance in the solar and thermal regions can now be expressed as:

$$
\begin{aligned}
& \bar{\Gamma}_{\text {solar }}=\frac{\bar{\tau}_{\text {solar }}^{\text {os }}}{1-\bar{r}_{\text {solar }}^{\text {oS }} \bar{r}_{\text {solar }}^{\text {S }}} \\
& \bar{\Gamma}_{\text {thermal }}=\frac{\bar{\tau}_{\text {thermal }}^{\text {os }}}{1-\bar{r}_{\text {thermal }}^{\text {So }} \bar{r}_{\text {thermal }}^{\text {is }}}
\end{aligned}
$$

Because the net cooling power calculated using either the spectral properties or effective properties needs to be the same, we can express the effective properties of the outer and inner surfaces in the solar and thermal regions of the spectrum as:

$$
\begin{aligned}
& \bar{\alpha}_{\text {solar }}^{\text {os }}=\frac{\int_{0}^{\lambda_{o}} I_{\text {solar }}(\lambda) \varepsilon_{o S}(\lambda)\left(1+\Gamma(\lambda) r_{i s}(\lambda)\right) d \lambda-\int_{0}^{\lambda_{o}} \varepsilon_{o S}(\lambda)\left(1+\Gamma(\lambda) r_{i s}(\lambda)\right) \frac{C_{1}}{\lambda^{5}}\left[\frac{1}{\exp \left(C_{2} / T_{o s} \lambda\right)-1}-\frac{\varepsilon_{\text {sky }}(\lambda)}{\exp \left(C_{2} / T_{s k y} \lambda\right)-1}\right] d \lambda}{\left(1+\bar{\Gamma}_{\text {solar }} \bar{r}_{\text {solar }}^{o S}\right)\left(\int_{0}^{\lambda_{o}} I_{\text {solar }}(\lambda) d \lambda-\int_{0}^{\lambda} \frac{C_{1}}{\lambda^{5}}\left[\frac{1}{\exp \left(C_{2} / T_{o s} \lambda\right)-1}-\frac{\varepsilon_{s k y}(\lambda)}{\exp \left(C_{2} / T_{s k y} \lambda\right)-1}\right] d \lambda\right)} \\
& \bar{\varepsilon}_{\text {thermal }}^{\text {os }}=\frac{\int_{\lambda_{o}}^{\infty} I_{\text {solar }}(\lambda) \varepsilon_{o S}(\lambda)\left(1+\Gamma(\lambda) r_{i s}(\lambda)\right) d \lambda-\int_{\lambda_{o}}^{\infty} \varepsilon_{o s}(\lambda)\left(1+\Gamma(\lambda) r_{i s}(\lambda)\right) \frac{C_{1}}{\lambda^{5}}\left[\frac{1}{\exp \left(C_{2} / T_{o s} \lambda\right)-1}-\frac{\varepsilon_{s k y}(\lambda)}{\exp \left(C_{2} / T_{s k y} \lambda\right)-1}\right] d \lambda}{\left(1+\bar{\Gamma}_{\text {thermal }} \bar{r}_{\text {thermal }}\right)\left(\int_{\lambda_{o}}^{\infty} I_{\text {solar }}(\lambda) d \lambda-\int_{\lambda_{o}}^{\infty} \frac{C_{1}}{\lambda^{5}}\left[\frac{1}{\exp \left(C_{2} / T_{o s} \lambda\right)-1}-\frac{\varepsilon_{s k y}(\lambda)}{\exp \left(C_{2} / T_{s k y} \lambda\right)-1}\right] d \lambda\right)}
\end{aligned}
$$


$\bar{\alpha}_{\text {solar }}^{\text {is }}=\frac{\int_{0}^{\lambda_{o}} I_{\text {solar }}(\lambda) \varepsilon_{i s}(\lambda) \Gamma(\lambda) d \lambda-\int_{0}^{\lambda_{o}} \varepsilon_{i s}(\lambda) \Gamma(\lambda) \frac{C_{1}}{\lambda^{5}}\left[\frac{1}{\exp \left(C_{2} / T_{i s} \lambda\right)-1}-\frac{\varepsilon_{s k y}(\lambda)}{\exp \left(C_{2} / T_{s k y} \lambda\right)-1}\right] d \lambda}{\bar{\Gamma}_{\text {solar }}\left(\int_{0}^{\lambda_{o}} I_{\text {solar }}(\lambda) d \lambda-\int_{0}^{\lambda o} \frac{C_{1}}{\lambda^{5}}\left[\frac{1}{\exp \left(C_{2} / T_{o s} \lambda\right)-1}-\frac{\varepsilon_{s k y}(\lambda)}{\exp \left(C_{2} / T_{s k y} \lambda\right)-1}\right] d \lambda\right)}$
$\bar{\varepsilon}_{\text {thermal }}^{\text {is }}=\frac{\int_{\lambda_{o}}^{\infty} I_{\text {solar }}(\lambda) \varepsilon_{\text {is }}(\lambda) \Gamma(\lambda) d \lambda-\int_{\lambda_{o}}^{\infty} \varepsilon_{i s}(\lambda) \Gamma(\lambda) \frac{C_{1}}{\lambda^{5}}\left[\frac{1}{\exp \left(C_{2} / T_{o s} \lambda\right)-1}-\frac{\varepsilon_{s k y}(\lambda)}{\exp \left(C_{2} / T_{s k y} \lambda\right)-1}\right] d \lambda}{\bar{\Gamma}_{\text {thermal }}\left(\int_{\lambda_{o}}^{\infty} I_{\text {solar }}(\lambda) d \lambda-\int_{\lambda_{o}{ }^{2}}^{\infty} \bar{\lambda}_{1}\left[\frac{1}{\exp \left(C_{2} / T_{o s} \lambda\right)-1}-\frac{\varepsilon_{s k y}(\lambda)}{\exp \left(C_{2} / T_{s k y} \lambda\right)-1}\right] d \lambda\right)}$

Also, using Kirchhoff's law of thermal radiation, we can write:

$\bar{\alpha}_{\text {solar }}^{\text {os }}+\bar{r}_{\text {solar }}^{\text {os }}+\bar{\tau}_{\text {solar }}^{\text {os }}=1$

$\bar{\varepsilon}_{\text {thermal }}^{\text {os }}+\bar{r}_{\text {thermal }}^{\text {os }}+\bar{\tau}_{\text {thermal }}^{\text {os }}=1$,

$\bar{\alpha}_{\text {solar }}^{i s}+\bar{r}_{\text {solar }}^{i s}=1$,

$\bar{\varepsilon}_{\text {thermal }}^{\text {is }}+\bar{r}_{\text {thermal }}^{i s}=1$

Note that there are ten variables $\left(\bar{\alpha}_{\text {solar }}^{\text {os }}, \bar{r}_{\text {solar }}^{\text {os }}, \bar{\tau}_{\text {solar }}^{\text {os }}, \bar{\varepsilon}_{\text {thermal }}^{\text {os }}, \bar{r}_{\text {thermal }}^{\text {os }}, \bar{\tau}_{\text {thermal }}^{\text {os }}, \bar{\alpha}_{\text {solar }}^{\text {is }}\right.$, $\bar{r}_{\text {solar }}^{i s}, \bar{\varepsilon}_{\text {thermal }}^{i s}$, and $\bar{r}_{\text {thermal }}^{\text {is }}$ ) but only eight equations (24-31), which means that these equations cannot be solved. Fortunately, commonly used inner substrate surfaces on building facades have nearly spectrally independent radiative properties, which means $\bar{\alpha}_{\text {solar }}^{i s}, \bar{r}_{\text {solar }}^{\text {is }}, \bar{\varepsilon}_{\text {thermal }}^{\text {is }}$, and $\bar{r}_{\text {thermal }}^{\text {is }}$ are known and nearly constant, reducing variable as well as equation count (24-29) to six. Lastly, the equations describing the effective properties are non-linear; therefore, we cannot obtain these variables directly but need an iterative numerical solver. One of such solver schemes written in MATLAB is provided in the supplementary information.

\subsection{Iterative Solver}

In this study, we employed MATLAB's inbuilt optimization function fmincon to find the minimum constrained nonlinear multivariable function $\left(\left|Q_{n e t}^{\text {spectral }}-Q_{\text {net }}^{\text {effective }}\right|\right)$, which is the absolute difference in the net radiation cooling obtained using spectral and effective properties. We assigned the radiative surface parameters with an initial estimate of 0.5 , lower bound of 0 , and upper bound of 1 . We used an optimality tolerance of $10^{-6}$, function tolerance of $10^{-6}$, and step tolerance of $10^{-10}$ as the threshold values to stop the iterations, if crossed. Additional details about the function and the tolerances can be found in the references ${ }^{24,25}$. 


\section{Results and Discussion}

To verify and validate our predictions of effective property values, we performed several case studies comprising radiative surfaces with the ideal broadband properties shown in Figure 3(a), the ideal selective properties depicted in Figure 3(b), the realistic diffuse properties with no transmissivity shown in Figure 3(c), and the realistic diffuse properties with transmissivity shown in Figure 3(d).
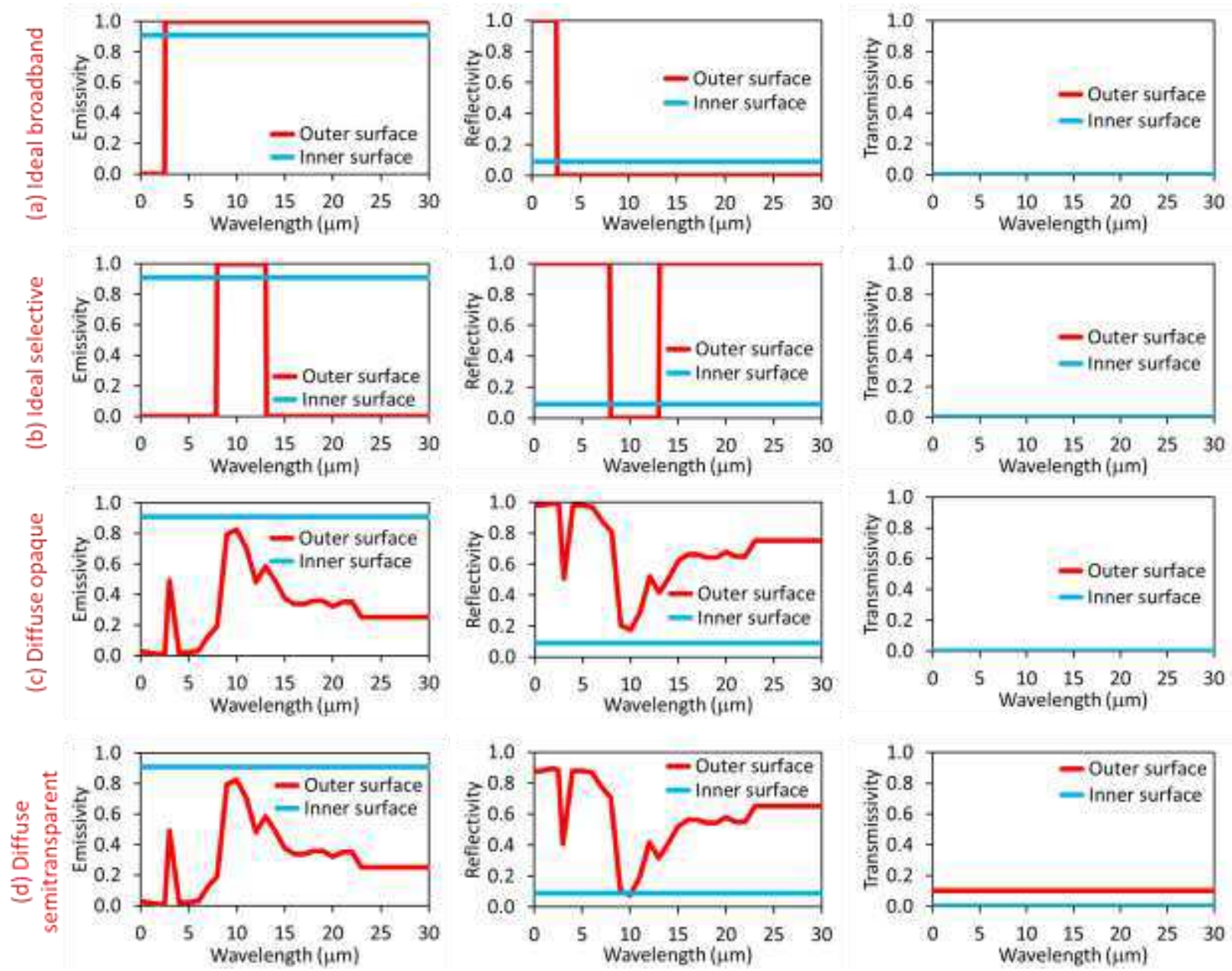

Figure 3. Radiative cooling surfaces with (a) ideal broadband properties, (b) ideal selective properties, (c) realistic diffuse properties with no transmissivity, and (d) realistic diffuse properties with $10 \%$ transmissivity. The inner surface properties are considered constant and realistic, the only the outer surface properties vary in the different cases.

We first calculated the value of the effective properties of the radiative surfaces using the traditional method described in Section 2.2, and then using the developed method explained in 
Section 2.3. We compared the net radiative cooling at different surface temperatures using spectrally distributed properties and the effective properties obtained from the two methods. Table 1 shows the various parameters used for the calculations, and Figure 4 depicts the wavelengthdependent solar power obtained using blackbody radiation and sky emissivity obtained from the sky transmission files at reference ${ }^{26}$. The realistic spectrally varying emissivity was obtained from reference ${ }^{15}$. Lastly, we computed the estimation error, defined as the sum of absolute differences between the heat fluxes in solar and thermal regions at the two surfaces, and percentage error as the estimation error over net radiation cooling obtained using spectrally distributed properties.

Error $=\left|Q_{\text {net }, 1}^{\text {spectral }}-Q_{\text {net }, 1}^{\text {effective }}\right|_{\text {os }}+\left|Q_{\text {net }, 2}^{\text {spectral }}-Q_{\text {net }, 2}^{\text {effective }}\right|_{\text {os }}+\left|Q_{\text {net }, 1}^{\text {spectral }}-Q_{\text {net }, 1}^{\text {effective }}\right|_{i s}+$ $\left|Q_{\text {net }, 2}^{\text {spectral }}-Q_{\text {net }, 2}^{\text {effective }}\right|_{\text {is }}$

$\%$ error $=\frac{\text { Error }}{Q_{\text {net }, 1}^{\text {spectral }}+Q_{\text {net }, 2}^{\text {spectral }}} \times 100$

where subscript 1 and 2 denote solar and thermal regimes at cut-off wavelength as $2.5 \mu \mathrm{m}$, and superscript "spectral" and "effective" represent radiations calculated using spectral and effective radiative properties.

Table 1. Constant and variable parameters used for the radiation cooling calculations.

\begin{tabular}{lcc}
\hline \multicolumn{1}{c}{ Parameter } & Unit & Value \\
\hline Solar surface temperature & $\mathrm{K}$ & 5778 \\
Radiative surface temperature & $\mathrm{K}$ & $250-300$ \\
Sky temperature & $\mathrm{K}$ & 250 \\
$C_{1}=2 \pi h c^{2}$ & $\mathrm{~W}-\mu \mathrm{m}^{4} / \mathrm{m}^{2}$ & $3.74 \times 10^{8}$ \\
$C_{2}=h c / k_{B}$ & $\mu \mathrm{m}-\mathrm{K}$ & $1.44 \times 10^{4}$ \\
Stephan Boltzmann constant & $\mathrm{W}-\mathrm{K} / \mathrm{m}^{2}$ & $5.67 \times 10^{4}$ \\
Total solar power & $\mathrm{W} / \mathrm{m}^{2}$ & 961 \\
\hline
\end{tabular}


(a)

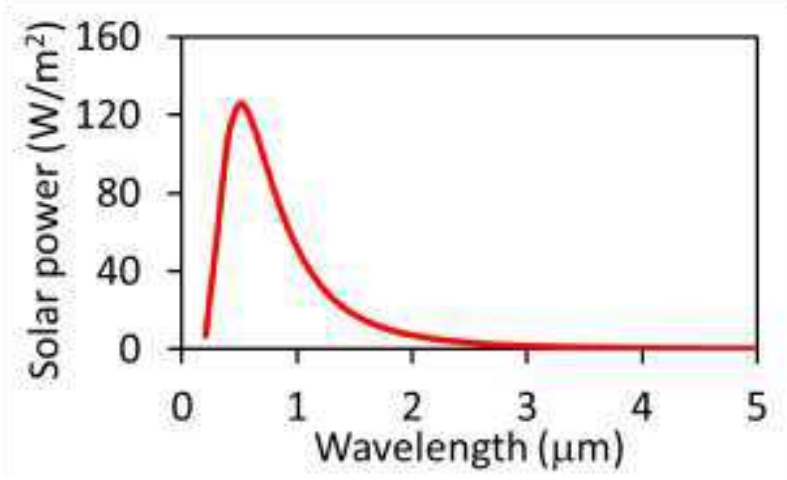

(b)

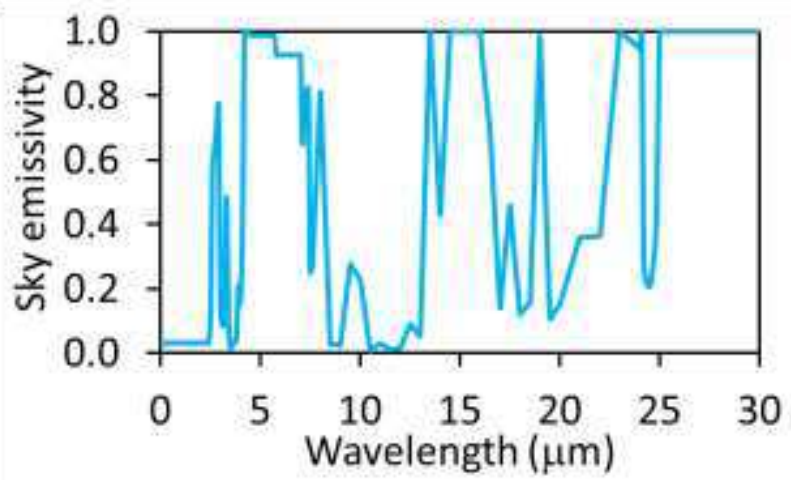

Figure 4. Spectrally distributed solar power obtained using blackbody radiation and sky emissivity taken from the sky transmission files. ${ }^{26}$

\subsection{Spectral Versus Effective Parameters Using the Traditional Method}

Figure 5 shows the net radiation cooling calculated using spectral properties and effective properties obtained using the traditional method. The net radiation cooling is calculated at different surface temperatures between $250 \mathrm{~K}$ and $300 \mathrm{~K}$, and the sky temperature is fixed at $250 \mathrm{~K}$. The traditional method relies on averaging each surface parameter locally, instead of the net cooling effect. This method, therefore, works very well when the surface parameters remain nearly unchanged. For instance, in the case of ideal broadband, the surface properties are constant in the solar and thermal regimes. Consequently, the error between the net radiation cooling obtained using spectral and effective properties is small. More specifically, as shown in Figure 5(a), the root mean square error (RMSE) in this case is only $1.5 \%$.

In other cases, however, when surface properties change with wavelength, effective properties obtained from traditional methods are not accurate. For instance, in the case of an ideal selective surface, radiative properties undergo a step change near the atmospheric transparency window (Figure 5(b)). The difference between net cooling obtained from spectral properties and effective properties is 30\%-65\% and the RMSE is close to 47\%. As shown in Figure 5(c), in the case of the diffuse opaque surface having a realistic spectral dependent emissivity, the error in the net cooling calculation by effective properties is $8 \%-50 \%$, with an RMSE of $26.3 \%$. The relative error in the calculation further increases if the coating surface is semitransparent (Figure 5(d)); the root mean square error in this case is $71.1 \%$, despite the fact that the transmissivity of the coating is only 0.1 . 
It is interesting to note that the net radiation cooling decreases from Figure 5(a) to Figure 5(d). The ideal broadband emissivity provides maximum cooling, up to $\sim 300 \mathrm{~W} / \mathrm{m}^{2}$ at a surface temperature of $300 \mathrm{~K}$. The ideal spectrally selective emissivity results in the net cooling of around $142 \mathrm{~W} / \mathrm{m}^{2}$ at surface temperature of $300 \mathrm{~K}$. The emissivity of a realistic surface is neither ideal broadband nor ideal spectrally selective, leading to lower radiation cooling than the ideal cases. We note the net cooling of around $127 \mathrm{~W} / \mathrm{m}^{2}$ at surface temperature of $300 \mathrm{~K}$. In the case where the semitransparent coating has a small transmissivity of $10 \%$ added over a highly absorptive substrate surface $(\alpha=0.91)$, the net radiation cooling decreased dramatically. Note that there is no cooling, but heating, until the temperature difference between surface and space is around $30 \mathrm{~K}$. When the surface temperature is $300 \mathrm{~K}$, we observed net radiative cooling of $\sim 65 \mathrm{~W} / \mathrm{m}^{2}$.
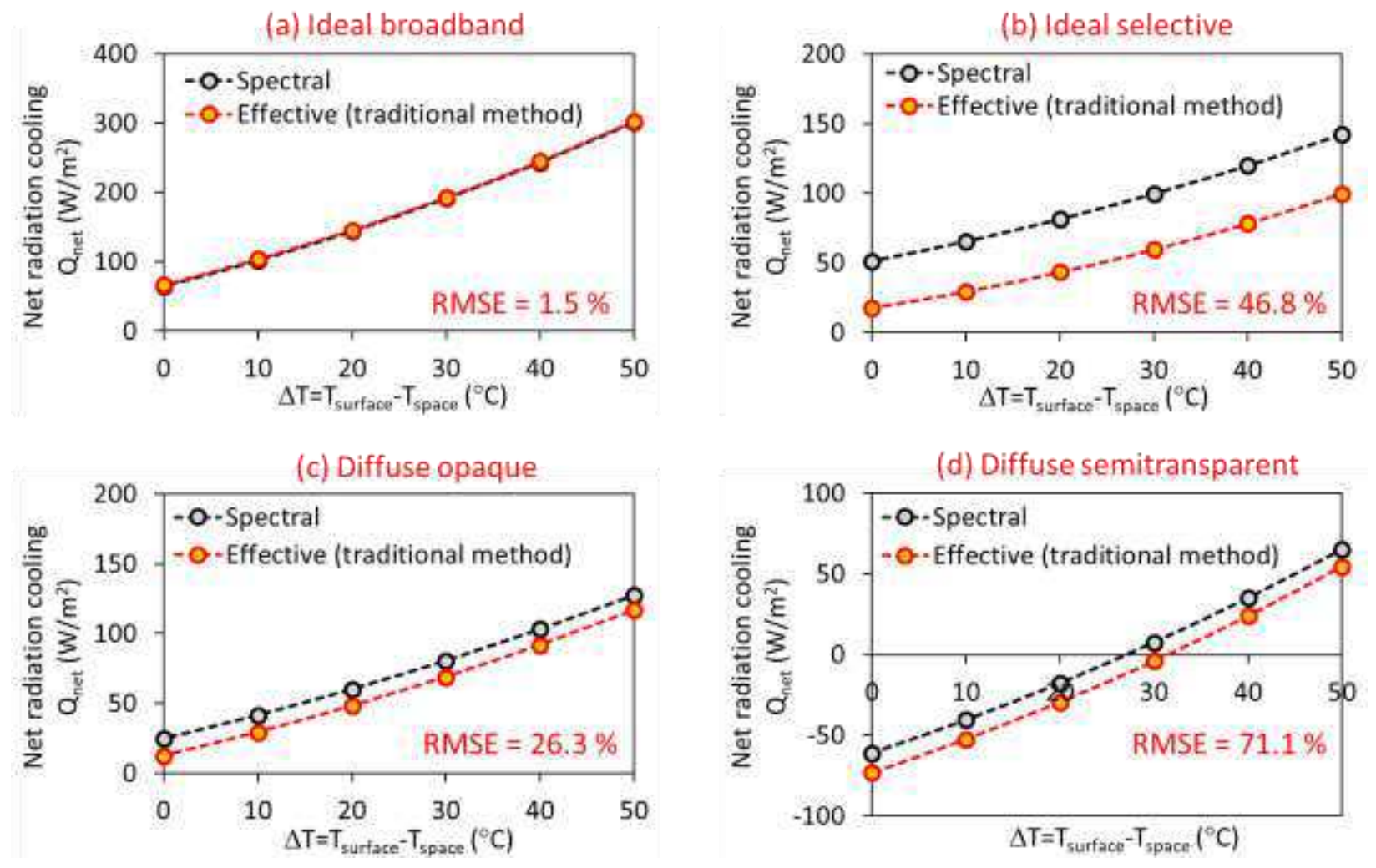

Figure 5. The net radiation cooling calculated using spectral properties and effective properties obtained using the traditional method: (a) ideal broadband emissivity, (b) ideal selective emissivity, (c) diffuse opaque with realistic spectrally varying emissivity, and (d) diffuse surface with semitransparent coating. 


\subsection{Spectral Versus Effective Parameters Using the Proposed Method}

Figure 6 compares the net radiation cooling calculated using the effective properties obtained from the proposed method versus the spectral properties. The differences are very small. In fact, the RMSE in all the cases is less than $0.2 \%$. This is a substantial improvement over the radiation cooling estimation using the traditional effective method. Again, the net cooling is highest in the case of ideal broadband surface, followed by ideal selective, diffuse opaque, and lastly the diffuse semitransparent surface.
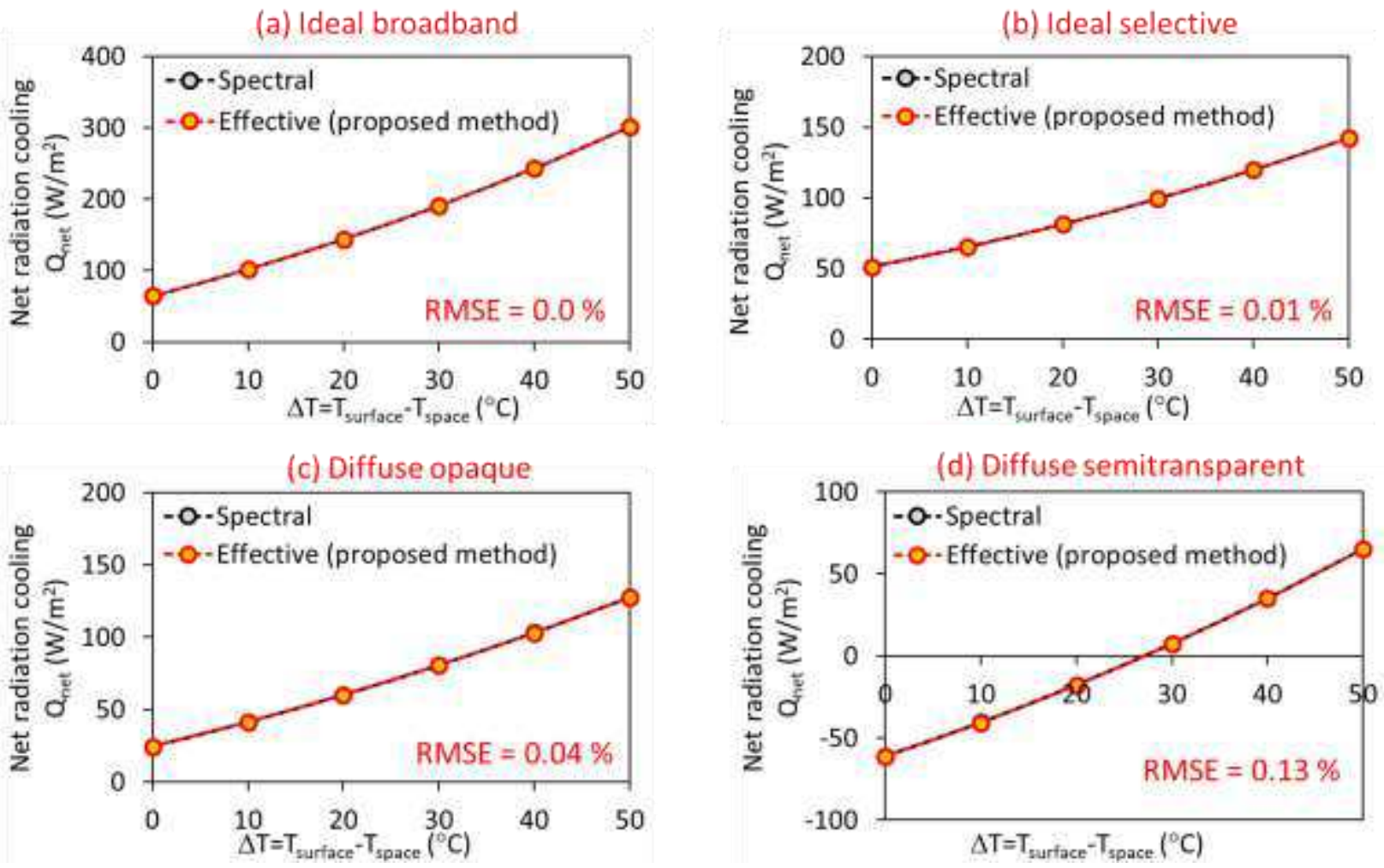

Figure 6. The net radiation cooling calculated using spectral properties and effective properties obtained using the proposed method: (a) ideal broadband emissivity, (b) ideal selective emissivity, (c) diffuse opaque with realistic spectrally varying emissivity, and (d) diffuse surface with semitransparent coating.

Table 2 lists the net radiation cooling at different surface temperatures calculated using the two effective methods. Because the radiation cooling generated by the semitransparent coating is significantly lower than that of the opaque coating, the transmissivity of the radiative coating and 
the solar absorption by the substrate surface cannot be ignored, particularly when the solar absorptivity of the substrate is large. The thin radiative coating over the asphalt roof shingles can be a classic example. The asphalt shingles have a solar absorptivity of 0.91 , which can substantially reduce the net radiation cooling generated by the radiative coating.

Table 2. Comparison of the net radiation cooling calculated using spectral and effective properties.

\begin{tabular}{ccccc}
\hline $\begin{array}{c}\text { Surface temperature } \\
(\mathrm{K})\end{array}$ & Radiative surface & Spectral & Traditional & Proposed \\
& & $\left(\mathrm{W} / \mathrm{m}^{2}\right)$ & effective & effective \\
& & & $\left(\mathrm{W} / \mathrm{m}^{2}\right)$ & $\left(\mathrm{W} / \mathrm{m}^{2}\right)$ \\
\hline \multirow{2}{*}{$250 \mathrm{~K}$} & Ideal broadband & 64.6 & 66.4 & 64.6 \\
& Ideal selective & 51.3 & 17.8 & 51.3 \\
& Diffuse opaque & 24.5 & 12.3 & 24.5 \\
& Diffuse semitransparent & -61.1 & -73.1 & -61.1 \\
\hline \multirow{2}{*}{$300 \mathrm{~K}$} & Ideal broadband & 301.0 & 302.4 & 301.0 \\
& Ideal selective & 142.5 & 99.6 & 142.5 \\
& Diffuse opaque & 127.4 & 116.6 & 127.4 \\
& Diffuse semitransparent & 65.2 & 54.8 & 65.1 \\
\hline
\end{tabular}

\subsection{Comparison With Commercial Finite Element Code Results}

To increase our confidence in the proposed effective method, we employed an established commercial finite element code, COMSOL Multiphysics. We performed three set of simulations: first using the spectral properties, second using the effective properties from the traditional method, and third using the effective properties from the proposed method. It is important to highlight that the spectral simulations in COMSOL Multiphysics are not truly spectral because the program only allows users to input surface properties in multiple bands instead of a continually varying data set. We used nearly 40 spectral bands, each having bandwidth of $0.5 \mu \mathrm{m}$; radiation outside of these 
bands are assumed constant. This deviation resulted in some differences in the calculated versus simulated results. However, overall, as shown in Figure 7, the difference in the modeling results using the proposed effective method is much smaller $(<6 \%)$ compared to the modeling results using the traditional effective method (error up to $36 \%$ ).

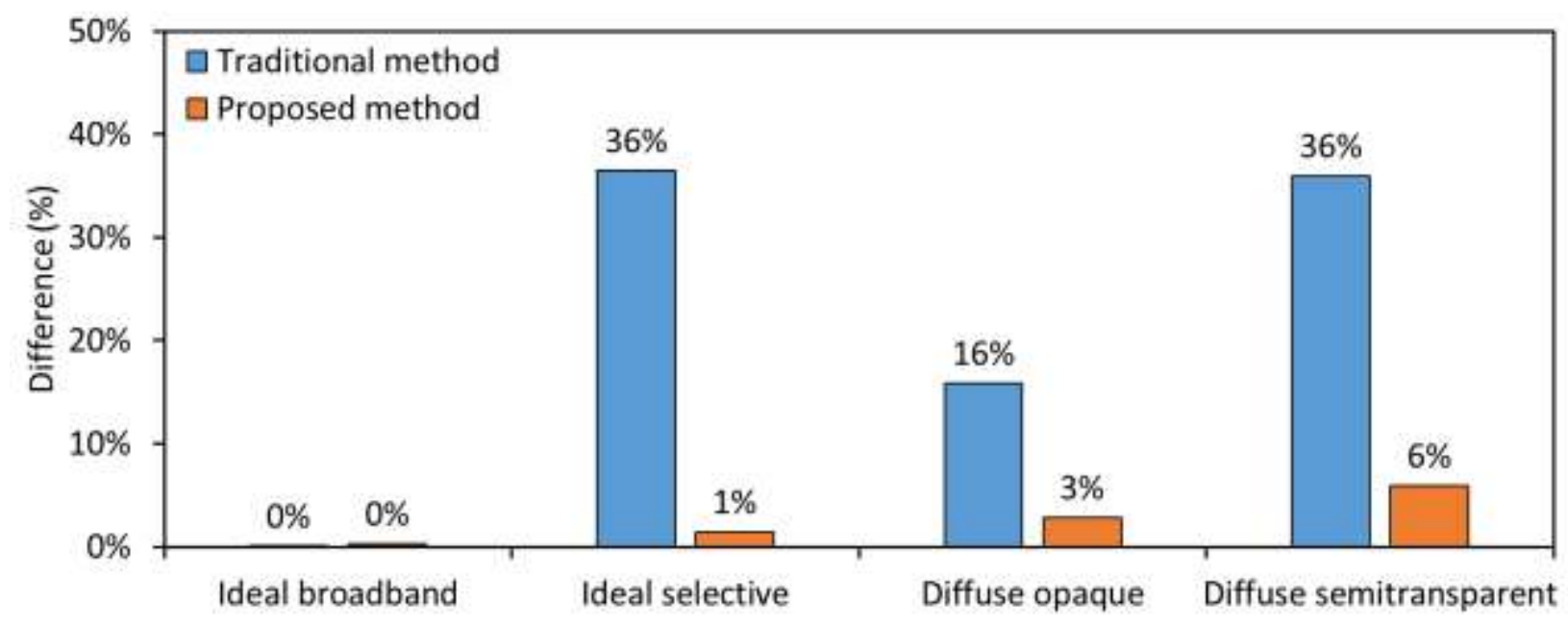

Figure 7. Comparison using a commercial finite element code, COMSOL Multiphysics. Differences in the net radiation cooling simulated using effective properties obtained from the traditional and proposed methods.

\section{Conclusions}

In this paper, we provide an iterative method to estimate the effective surface radiative properties in solar and thermal regions of the spectrum for accurate estimation of net radiation cooling. We compared the effectiveness of the proposed method over the traditional averaging method and noted a significant improvement. Whereas the traditional method works mainly for materials whose properties are weak functions of wavelength, such as the ideal broadband surface properties, the proposed method accurately estimates radiation cooling even in the cases when surface properties are strongly wavelength dependent. The root mean square error in the radiation cooling calculations using the proposed effective method was less than $0.2 \%$, in comparison to the error up to $71 \%$ from the traditional effective method. We also compared the results of the proposed method with results generated by a commercial finite element code, COMSOL Multiphysics, and noted maximum difference of less than $6 \%$ in the case of the proposed effective method against that of up to $36 \%$ in the case of the traditional effective method. We have also provided the 
MATLAB code in the supplementary information, which can be further customized for other applications.

\section{Acknowledgements}

This work was authored by the National Renewable Energy Laboratory (NREL), operated by Alliance for Sustainable Energy, LLC, for the U.S. Department of Energy (DOE) under Contract No. DE-AC36-08GO28308. This work was supported by the Wells Fargo Innovation Incubator (IN2) program funded by the Wells Fargo Foundation and co-administered by NREL. The views expressed in the article do not necessarily represent the views of the DOE or the U.S. Government. The U.S. Government retains and the publisher, by accepting the article for publication, acknowledges that the U.S. Government retains a nonexclusive, paid-up, irrevocable, worldwide license to publish or reproduce the published form of this work, or allow others to do so, for U.S. Government purposes.

\section{References}

1 Zhao, D. et al. Radiative sky cooling: Fundamental principles, materials, and applications. Applied Physics Reviews 6, 021306 (2019).

2 Sun, X., Sun, Y., Zhou, Z., Alam, M. A. \& Bermel, P. Radiative sky cooling: fundamental physics, materials, structures, and applications. Nanophotonics 6, 997-1015 (2017).

3 Gentle, A. R. \& Smith, G. B. Radiative heat pumping from the earth using surface phonon resonant nanoparticles. Nano letters 10, 373-379 (2010).

4 Mandal, J. et al. Hierarchically porous polymer coatings for highly efficient passive daytime radiative cooling. Science 362, 315-319 (2018).

5 Hsu, P.-C. et al. Radiative human body cooling by nanoporous polyethylene textile. Science 353, 1019-1023 (2016).

6 Orel, B., Gunde, M. K. \& Krainer, A. Radiative cooling efficiency of white pigmented paints. Solar energy 50, 477-482 (1993).

7 Nilsson, T. M. J. \& Niklasson, G. A. Radiative cooling during the day: simulations and experiments on pigmented polyethylene cover foils. Solar energy materials and solar cells 37, 93-118 (1995).

8 Granqvist, C. G., Hjortsberg, A. \& Eriksson, T. S. Radiative cooling to low temperatures with selectivity IR-emitting surfaces. Thin Solid Films 90, 187-190 (1982).

9 Granqvist, C. G. \& Hjortsberg, A. Surfaces for radiative cooling: Silicon monoxide films on aluminum. Applied Physics Letters 36, 139-141 (1980).

10 Eicker, U. \& Dalibard, A. Photovoltaic-thermal collectors for night radiative cooling of buildings. Solar Energy 85, 1322-1335 (2011).

11 Meir, M. G., Rekstad, J. B. \& LØvvik, O. M. A study of a polymer-based radiative cooling system. Solar energy 73, 403-417 (2002). 
12 Granqvist, C. G. \& Hjortsberg, A. Radiative cooling to low temperatures: General considerations and application to selectively emitting SiO films. Journal of Applied Physics 52, 4205-4220 (1981).

13 Catalanotti, S. et al. The radiative cooling of selective surfaces. Solar Energy 17, 83-89 (1975).

14 Rephaeli, E., Raman, A. \& Fan, S. Ultrabroadband photonic structures to achieve highperformance daytime radiative cooling. Nano letters 13, 1457-1461 (2013).

15 Raman, A. P., Abou Anoma, M., Zhu, L., Rephaeli, E. \& Fan, S. Passive radiative cooling below ambient air temperature under direct sunlight. Nature 515, 540-544 (2014).

16 Chen, L. et al. Sub-ambient radiative cooling and its application in buildings. Building Simulation 13, 1165-1189 (2020).

17 Jeon, S. \& Shin, J. Ideal spectral emissivity for radiative cooling of earthbound objects. Scientific reports 10, 1-7 (2020).

18 Fu, Y., Yang, J., Su, Y. S., Du, W. \& Ma, Y. G. Daytime passive radiative cooler using porous alumina. Solar Energy Materials and Solar Cells 191, 50-54 (2019).

19 Bao, H. et al. Double-layer nanoparticle-based coatings for efficient terrestrial radiative cooling. Solar Energy Materials and Solar Cells 168, 78-84 (2017).

20 Chen, M. et al. A pragmatic and high-performance radiative cooling coating with nearideal selective emissive spectrum for passive cooling. Coatings 10, 144 (2020).

21 Hossain, M. M. \& Gu, M. Radiative cooling: principles, progress, and potentials. Advanced Science 3, 1500360 (2016).

22 Papadakis, G. et al. Review Paper (SE-Structures and Environment): Radiometric and thermal properties of, and testing methods for, greenhouse covering materials. Journal of Agricultural Engineering Research 77, 7-38 (2000).

23 Mitts, S. J. \& Smith, T. F. Solar energy transfer through semitransparent plate systems. Journal of thermophysics and heat transfer 1, 307-312 (1987).

24 MathWorks. fmincon: https://www.mathworks.com/help/optim/ug/fmincon.html. Accessed on 06-28-2021.

25 MathWorks. Tolerances and Stopping Criteria: https://www.mathworks.com/help/optim/ug/tolerances-and-stopping-criteria.html. Accessed on 06-28-2021.

26 Lord, S. D. NASA Technical Memorandum 103957. 1992. https://www.gemini.edu/observing/telescopes-and-sites/sites\#Transmission. Accessed on 06-28-2021. 


\section{Supplementary Files}

This is a list of supplementary files associated with this preprint. Click to download.

- Supplementaryinformation08022021.docx 